\title{
ZERO-DIVISOR GRAPHS OF POLYNOMIALS AND POWER SERIES OVER COMMUTATIVE RINGS
}

\author{
M. AXTELL, J. COYKENDALL, AND J. STICKLES
}

\begin{abstract}
We recall several results of zero divisor graphs of commutative rings. We then examine the preservation of diameter and girth of the zero divisor graph under extension to polynomial and power series rings.
\end{abstract}

The concept of the graph of the zero-divisors of a ring was first introduced by Beck in [3] when discussing the coloring of a commutative ring. In his work all elements of the ring were vertices of the graph. D.D. Anderson and Naseer used this same concept in [1]. We adopt the approach used by D.F. Anderson and Livingston in [2] and consider only nonzero zero-divisors as vertices of the graph. In the first section we provide the reader with a few known results concerning the girth and diameter of the graph of zero-divisors of a commutative ring. In the second section we examine the preservation and lack thereof of the diameter of the zero-divisor graphs under extensions to polynomial and power series rings, while in the third section we consider the effects of the same extensions upon the girth of the graphs.

Throughout, $R$ is a commutative ring and, unless otherwise stated, is assumed to have an identity. We use $Z(R)$ to denote the set of zerodivisors of $R$; we use $Z^{*}(R)$ to denote the set of nonzero zero-divisors of $R$. By the zero-divisor graph of $R$, denoted $\Gamma(R)$, we mean the graph whose vertices are the nonzero zero-divisors of $R$, and for distinct $r, s \in Z^{*}(R)$, there is an edge connecting $r$ and $s$ if and only if $r s=0$. For two distinct vertices $a$ and $b$ in a graph $\Gamma$, the distance between $a$ and $b$, denoted $d(a, b)$, is the length of the shortest path connecting $a$ and $b$, if such a path exists; otherwise, $d(a, b)=\infty$. The diameter of a graph $\Gamma$ is $\operatorname{diam}(\Gamma)=\sup \{d(a, b) \mid a$ and $b$ are distinct vertices of $\Gamma\}$. We will use the notation $\operatorname{diam}(R)$ and $\operatorname{diam}(\Gamma(R))$ interchangeably to denote the diameter of the graph of $Z^{*}(R)$. The girth of a graph $\Gamma$, denoted $g(\Gamma)$, is the length of the shortest cycle in $\Gamma$, provided $\Gamma$ contains a cycle; otherwise, $\mathrm{g}(\Gamma)=\infty$. We will use the notation $\mathrm{g}(R)$ and $\mathrm{g}(\Gamma(R))$ interchangeably to denote the girth of the graph of $Z^{*}(R)$. A graph is said to be connected if there exists a path between any two distinct vertices, and a graph is complete if it is connected 
with diameter one. We use the set notation $A^{*}$ to refer to the nonzero elements of $A$.

\section{Diameter and Girth of $\Gamma(R)$}

Anderson and Livingston first proved the following result in [2]:

Theorem 1. Let $R$ be a commutative ring, not necessarily with identity, with $Z^{*}(R) \neq \emptyset$. Then $\Gamma(R)$ is connected and $\operatorname{diam}(\Gamma(R)) \leq 3$.

Anderson and Livingston used this result combined with the graph theoretical result that for any graph $\Gamma$, if $\Gamma$ contains a cycle, then $\mathrm{g}(\Gamma) \leq 2 \cdot \operatorname{diam}(\Gamma)+1$ to conclude $\mathrm{g}(\Gamma(R)) \leq 7$. They conjectured $\mathrm{g}(\Gamma(R)) \leq 4$. Mulay proved this result in $[7]$. We provide an alternate proof below.

Theorem 2. Let $R$ be a commutative ring, not necessarily with identity. If $\Gamma(R)$ contains a cycle, then $\mathrm{g}(\Gamma(R)) \leq 4$.

Proof. If $\Gamma(R)$ contains a cycle $x_{0}-x_{1}-\cdots-x_{n}-x_{0}$ with $n \geq 4$ and $x_{i} x_{j}=0$ for some $i$ and $j$ where $j>i+1$ and either $0 \leq i<j \leq n-1$ or $1 \leq i<j \leq n$, we can eliminate $x_{k}$ where $i<k<j$ to obtain a shorter cycle. So, assume $\Gamma(R)$ contains a cycle $x_{0}-x_{1}-\cdots-x_{n}-x_{0}$ with $n \geq 4$ and $x_{i} x_{j} \neq 0$ for any $i$ and $j$ under the above conditions. Then, we can form a cycle $x_{0}-x_{1} x_{n-1}-x_{n}-x_{0}$ of length 3 .

So, assume, without loss of generality, $x_{1} x_{n-1}=x_{0}$. This necessarily forces $x_{0}^{2}=0$. If there exists $y \in R$ such that $x_{0} y \neq 0$ or $x_{0}$, then either $x_{0}-x_{1}-x_{0} y-x_{0}$ is a cycle of length 3 (if $x_{0} y \neq x_{1}$ ), or $x_{0}-x_{n}-x_{0} y-x_{0}$ is a cycle of length 3 (if $x_{0} y=x_{1}$ ). Finally, if $\left(x_{0}\right)=\left\{0, x_{0}\right\}$, then $\left(x_{0}\right) \subset\left(x_{1}\right)$, yielding a $y \in R$ such that $x_{1} y \notin\left\{0, x_{0}, x_{1}\right\}$. Now since $x_{0} x_{2} \neq 0, x_{1} y \neq x_{2}$. We get $x_{0}-x_{1}-x_{2}-x_{1} y-x_{0}$ is a cycle of length 4.

The upper bound in Theorem 2 can be achieved as seen by the ring $R=\mathbb{Z} \times \mathbb{Z}$.

\section{Diameter of $\Gamma(R), \Gamma(R[x])$ And $\Gamma(R[[x]])$}

It is an interesting question to consider the preservation of graph theoretic properties under various ring theoretic extensions. The first such extensions that come to mind are those of polynomial and power series extensions. Observe $\Gamma(R)$ is a subgraph of $\Gamma(R[x])$, which is a subgraph of $\Gamma(R[[x]])$. Also, it is straightforward to show that $\operatorname{diam}(\Gamma(R)) \leq$ $\operatorname{diam}(\Gamma(R[x]))$ and $\operatorname{diam}(\Gamma(R)) \leq \operatorname{diam}(\Gamma(R[[x]]))$

McCoy established that a polynomial $f(x)$ is a zero-divisor in $R[x]$ if and only if there is a nonzero element $r$ of $R$ with $r f(x)=0$ in 
[6]. Fields provided an analgous result for the power series ring over a Noetherian ring in [4]. In addition, Fields provides an example of a nonNoetherian ring and a zero-divisor power series that is not annihilated by any nonzero element of the original ring. We include Lemma 2 from [4] which will be used later in the paper.

Lemma 1. Let $R$ be a commutative ring with identity and let $f(x)=$ $\sum_{i=0}^{\infty} f_{i} x^{i} \in R[[x]]$. If, for some natural number $t, f_{t}$ is regular in $R$ while $f_{i}$ is nilpotent for $0 \leq i \leq t-1$, then $f(x)$ is regular in $R[[x]]$.

Theorem 3. If $R \neq \mathbb{Z}_{2} \times \mathbb{Z}_{2}$ then the following are equivalent:

(1) $\Gamma(R[[x]])$ is complete

(2) $\Gamma(R[x])$ is complete

(3) $\Gamma(R)$ is complete

Proof. Clearly, $(1) \Rightarrow(2) \Rightarrow(3)$. For $(3) \Rightarrow(1)$, since $R \neq \mathbb{Z}_{2} \times \mathbb{Z}_{2}$ we have by Theorem 2.8 of [2] that $x y=0$ for every $x, y \in Z^{*}(R)$. Therefore $\Gamma(R)$ complete implies $(Z(R))^{2}=0$. Let $f(x), g(x) \in Z^{*}(R[[x]])$. By Lemma 1, no coefficient of $f$ or $g$ can be regular since all non-regular coefficients of $f$ and $g$ are nilpotent. Thus all coefficients of $f$ and $g$ are zero-divisors in $R$. Since $\Gamma(R)$ complete, $f(x) g(x)=0$ and hence $\Gamma(R[[x]])$ is also complete.

If $R=\mathbb{Z}_{2} \times \mathbb{Z}_{2}$ then $\Gamma(R)$ is complete. However, $\Gamma(R[x])$ is not complete and hence $\Gamma(R[[x]])$ is not complete. To see this, let $a=$ $(1,0)+(1,0) x$ and $b=(1,0)+(1,0) x^{2}$. Then $a b \neq 0$ and $a-(0,1)-b$. Thus $\operatorname{diam}(\Gamma(R[x]))=\operatorname{diam}(\Gamma(R[[x]]))=2$.

Question: Is it possible to find a ring for which $\operatorname{diam}(\Gamma(R))=2$ but either $\operatorname{diam}(\Gamma(R[x]))=3$ or $\operatorname{diam}(\Gamma(R[[x]]))=3$ ? The following example from [4] provides a partial answer to this question.

Example 1. Let $K$ be a field, and let $R=K\left[Y,\{X\}_{i=0}^{\infty}\right] /\left(\left\{X_{0} Y\right\} \cup\right.$ $\left.\left\{X_{i}-X_{i+1} Y\right\}_{i=0}^{\infty}\right)$. Then, $\operatorname{diam}(\Gamma(R))=2$, but $\operatorname{diam}(\Gamma(R[[W]]))=3$.

Proof. In $R, \overline{X_{0}}$ annihilates all other variables, since $\overline{X_{0} Y}=0$ and $\overline{X_{0} X_{i}}=\overline{X_{0} X_{i+1} Y}=0$. In $R, \overline{X_{1}}$ annihilates all other variables but $\bar{Y}$, since $\overline{X_{1} X_{i}}=\overline{X_{1} X_{i+1} Y}=\overline{X_{0} X_{i+1}}=0$ but $\overline{X_{1} Y}=\overline{X_{0}} \neq 0$. In $R, \overline{X_{2}}$ annihilates all other variables but $\bar{Y}$, since $\overline{X_{2} X_{i}}=\overline{X_{2} X_{i+1} Y}=\overline{X_{1} Y}=$ 0 , but $\overline{X_{2} Y}=\overline{X_{1}} \neq 0$. Continuing this process we see that $\overline{X_{i}}(i \geq 1)$ annihilates all other variables besides $\bar{Y}$. Observe that $\overline{X_{i} Y}=\overline{X_{i-1}}$ and $\overline{X_{i} Y^{i+r}}=\overline{X_{0} Y^{r}}=0$ and $\overline{X_{i} Y^{i}}=\overline{X_{0}}$ and $\overline{X_{i+r} Y^{i}}=\overline{X_{r}}$. Thus, any element of $R$ can be written in the form $r=k_{0}+\sum_{j=1}^{\infty} l_{j} \bar{Y}^{j}+$ $\sum_{i=0}^{\infty} k_{i+1} \overline{X_{i}}$, where $k_{i}, l_{j} \in K$ and all but finitely many $k_{i}$ are zero; 
hence, $\operatorname{ann}(\bar{Y})=\left\{k \overline{X_{0}} \mid k \in K\right\}$. To see $\operatorname{diam}(\Gamma(R))=2$, notice that if $r \in Z(R)$, then $r$ has no constant term; hence $r_{1}-\overline{X_{0}}-r_{2}$ is a path of length 2 for any $r_{1}, r_{2} \in Z^{*}(R)$. To see $\operatorname{diam}(\Gamma(R[[W]]))=3$, let $f(W)=\bar{Y}-W$. Then $f \in Z^{*}(R[[W]])$ (see [4]). Let $g \in \operatorname{ann}(f)$. Without loss of generality, $g=a_{0}+a_{1} W+a_{2} W^{2}+\cdots$ with $a_{0} \neq 0$. Then, $(\bar{Y}-W) g=0 \Rightarrow a_{0} \bar{Y}=0 \Rightarrow a_{0}=k \overline{X_{0}}$. Further, $-k \overline{X_{0}} W+$ $a_{1} \bar{Y} W=0 \Rightarrow a_{1} \bar{Y}=k \overline{X_{0}} \neq 0$. Hence, $d(f, \bar{Y})>2$ so $d(f, \bar{Y})=3$.

The proof above also serves to show that $\operatorname{diam}(\Gamma(R[x]))=2$, since $\overline{X_{0}}$ annihilates all polynomial zero-divisors in $R[x]$. Thus this example also shows that $\operatorname{diam}(\Gamma(R[x]))=2$ need not imply $\operatorname{diam}(\Gamma(R[[x]]))=2$.

We remark that if $R$ is commutative with identity, then the set of regular elements of $R$ form a saturated and multiplicatively closed subset of $R$. Hence the collection of zero-divisors of $R$ is the set-theoretic union of prime ideals. We write $Z(R)=\bigcup_{i \in \Lambda} \mathcal{P}_{i}$ with $\mathcal{P}_{i}$ prime. We will also assume that these primes are maximal with respect to being contained in $Z(R)$.

Proposition 1. Let $R$ be a commutative ring with $\operatorname{diam}(\Gamma(R)) \leq 2$ and let $Z(R)=\bigcup_{i \in \Lambda} \mathcal{P}_{i}$. If there is an element in $Z(R)$ that is contained in a unique maximal $\mathcal{P}_{i}$ then $|\Lambda| \leq 2$.

Proof. We choose our element $p_{1} \in \mathcal{P}_{1}$ such that $p_{1}$ is contained in none of the other maximal primes in $Z(R)$. Assume that we have at least two other maximal primes which we denote as $\mathcal{P}_{2}$ and $\mathcal{P}_{3}$. Using Theorem 81 of [5], we choose $p_{2} \in \mathcal{P}_{2} \backslash\left(\mathcal{P}_{1} \cup \mathcal{P}_{3}\right)$. Note that the ideal $\left(p_{1}, p_{2}\right)$ is contained in no maximal prime ideal in $Z(R)$, hence there must be a regular element in $\left(p_{1}, p_{2}\right)$. We write $a p_{1}+b p_{2}=r$ with $r$ a regular element. Note that if there is a mutual annihilator of $p_{1}$ and $p_{2}$, then this element must annihilate $r$ and hence is 0 , since $r$ is regular. Since the diameter of $\Gamma(R)$ is 2 , this means that $p_{1} p_{2}=0 \in \mathcal{P}_{3}$. But since $p_{1} \notin \mathcal{P}_{3}$, this means that $p_{2} \in \mathcal{P}_{3}$ contradicting the choice of $p_{2}$. This establishes the proposition.

Here is a corollary that highlights the applications we have in mind.

Corollary 1. Let $R$ be a ring with $\operatorname{diam}(\Gamma(R)) \leq 2$ and let $Z(R)=$ $\bigcup_{i \in \Lambda} \mathcal{P}_{i}$. If $\Lambda$ is a finite set (in particular if $R$ is Noetherian), then $|\Lambda| \leq 2$.

Proof. We first remark that Theorem 80 of [5] shows that any Noetherian ring satisfies the hypothesis of the corollary. We will apply the previous result after showing that in $Z(R)$ there is an element that is contained in a unique maximal prime. Suppose that $Z(R)=\mathcal{P}_{1} \cup \mathcal{P}_{2} \cup \cdots \cup \mathcal{P}_{n}$ and suppose that every element of $\mathcal{P}_{1}$ 
is contained in one of the other maximal primes. This implies that $\mathcal{P}_{1} \backslash\left(\mathcal{P}_{2} \cup \mathcal{P}_{3} \cup \cdots \cup \mathcal{P}_{n}\right)=\emptyset$. Equivalently, we have $\mathcal{P}_{1} \subseteq \mathcal{P}_{2} \cup \mathcal{P}_{3} \cup \cdots \cup \mathcal{P}_{n}$. But by Theorem 81 of [5], this implies that $\mathcal{P}_{1} \subseteq \mathcal{P}_{i}$ for some $2 \leq i \leq n$, which contradicts the maximality of $\mathcal{P}_{1}$.

The next proposition is our final set-up for the Noetherian case.

Proposition 2. Let $R$ be a ring such that $\operatorname{diam}(\Gamma(R))=2$ and $Z(R)=$ $\mathcal{P}_{1} \cup \mathcal{P}_{2}$ with $\mathcal{P}_{1}$ and $\mathcal{P}_{2}$ distinct maximal primes in $Z(R)$. Then $\mathcal{P}_{1} \cap$ $\mathcal{P}_{2}=\{0\}$ (in particular, for all $p_{1} \in \mathcal{P}_{1}$ and $p_{2} \in \mathcal{P}_{2}, p_{1} p_{2}=0$ ).

Proof. We begin by verifying some annilihator relations of elements of $Z(R)$. Suppose first that $p_{1} \in \mathcal{P}_{1} \backslash \mathcal{P}_{2}$ and $p_{2} \in \mathcal{P}_{2} \backslash \mathcal{P}_{1}$. Since $\left(p_{1}, p_{2}\right)$ is not contained in either $\mathcal{P}_{1}$ or $\mathcal{P}_{2}$, there must be a regular element in $\left(p_{1}, p_{2}\right)$. Hence there is a linear combination of $p_{1}$ and $p_{2}$ that is regular, and hence $p_{1}$ and $p_{2}$ can possess no mutual annihilator. Since $\operatorname{diam}(\Gamma(R))=2$, we have that $p_{1} p_{2}=0$.

Now suppose that $p_{1} \in \mathcal{P}_{1} \backslash \mathcal{P}_{2}$ and $p_{2} \in \mathcal{P}_{2}$. If $p_{2}$ is not in $\mathcal{P}_{1}$ then $p_{1} p_{2}$ is immediately 0 . So we can assume that $p_{2} \in \mathcal{P}_{2} \cap \mathcal{P}_{1}$ and we select $y \in \mathcal{P}_{2} \backslash \mathcal{P}_{1}$. Note that $p_{2}+y \in \mathcal{P}_{2} \backslash \mathcal{P}_{1}$, and hence $p_{1}\left(p_{2}+y\right)=0=p_{1} p_{2}$.

We have shown that any element of $\mathcal{P}_{1} \backslash \mathcal{P}_{2}$ annihilates $\mathcal{P}_{2}$ (and of course, every element $\mathcal{P}_{2} \backslash \mathcal{P}_{1}$ annihilates $\left.\mathcal{P}_{1}\right)$. Suppose $x$ is a nonzero element of $\mathcal{P}_{1} \cap \mathcal{P}_{2}$. Note that the annihilator of $x$ necessarily contains all of $\mathcal{P}_{1} \backslash \mathcal{P}_{2}$ and $\mathcal{P}_{2} \backslash \mathcal{P}_{1}$. So the annililator of $(x) \subseteq Z(R)$ is contained in neither $\mathcal{P}_{1}$ nor $\mathcal{P}_{2}$, a contradiction.

Proposition 3. Let $R$ be a ring such that $\operatorname{diam}(\Gamma(R))=2$. If $Z(R)=$ $\mathcal{P}_{1} \cup \mathcal{P}_{2}$ is the union of precisely two maximal primes in $Z(R)$, then $\operatorname{diam}(R[x])=2$.

Proof. Let $f(x), g(x)$ be zero-divisors (with the degree of $f(x)$ greater than 0$)$. It is easy to see that it is necessary for $f(x)$ to be contained in $\mathcal{P}_{1}[x]$ or $\mathcal{P}_{2}[x]$ (indeed, if $a \in \mathcal{P}_{1} \backslash \mathcal{P}_{2}$ and $b \in \mathcal{P}_{2} \backslash \mathcal{P}_{1}$ are two coefficients of $f(x)$ then $a$ and $b$ must have a mutual annililator that is necessarily in $\mathcal{P}_{1} \cap \mathcal{P}_{2}=\{0\}$ by the previous result). There are two natural cases. If $f(x) \in \mathcal{P}_{1}[x]$ and $g(x) \in \mathcal{P}_{2}[x]$, then Proposition 2 shows that $f(x) g(x)=0$. On the other hand, if $f(x), g(x) \in \mathcal{P}_{1}[x]$, then any element of $\mathcal{P}_{2}$ suffices as a mutual annihilator. So $\operatorname{diam}(R[x])=$ 2 .

Surprisingly, the quasilocal case is a bit more problematic, but yields easily in the Noetherian case.

Proposition 4. Let $R$ be a Noetherian ring such that $\operatorname{diam}(\Gamma(R))=2$. If $Z(R)=\mathcal{P}$ is a prime ideal, then $\operatorname{diam}(R[x])=2$. 
Proof. By Theorem 82 in [5], $\mathcal{P}$ is annihilated by a single element (say $z$ ). Suppose that $f(x), g(x)$ are zero-divisors (with degree of $f(x)$ greater than 0$)$. If $f(x) g(x)=0$, we are done. If $f(x) g(x) \neq 0$, then neither $f(x)$ nor $g(x)$ is $z$, and so $z$ is a mutual annihilator of $f(x)$ and $g(x)$.

Theorem 4. If $R$ is Noetherian and $\operatorname{diam}(\Gamma(R))=2$, then $\operatorname{diam}(\Gamma(R[[x]]))=$ 2 .

Proof. By Corollary 1, either $Z(R)=\mathcal{P}_{1} \cup \mathcal{P}_{2}$ is the union of precisely two maximal primes in $Z(R)$ or $Z(R)=\mathcal{P}$ is a prime ideal. By Theorem 5 of [4], if $R$ is Noetherian, then $f(x) \in Z^{*}(R[[x]])$ if and only if $r f(x)=0$ for some nonzero $r \in R$. Thus, every coefficient of $f$ is a zero-divisor of $R$.

Assume $Z(R)=\mathcal{P}_{1} \cup \mathcal{P}_{2}$ is the union of precisely two maximal primes in $Z(R)$. If $f(x)=\sum f_{i} x^{i} \in Z^{*}(R[[x]])$, then by the proof of Proposition 3 we have that, without loss of generality, $f_{i} \in \mathcal{P}_{1}$ for all $i$. Hence every element of $\mathcal{P}_{2}$ will annihilate $f(x)$ by Proposition 2 . Thus $\operatorname{diam}(\Gamma(R[[x]]))=2$.

If $Z(R)=\mathcal{P}$ is a prime ideal, then by Theorem 82 in [5], $\mathcal{P}$ is annihilated by a single element (say $z$ ). The result follows in much the same way as the proof of Proposition 4.

The following theorem summarizes.

Theorem 5. Let $R$ be a ring such that $\operatorname{diam}(\Gamma(R))=2$ and $Z(R)$ is a finite union of primes. Then $Z(R)$ is necessarily the union of at most two primes. If $Z(R)$ is the union of two primes then $\operatorname{diam}(R[x])=2$, and additionally if $R$ is Noetherian, then $\operatorname{diam}(R[x])=\operatorname{diam}(R[[x]])=$ 2 .

Here is another observation.

Theorem 6. Let $R \neq \mathbb{Z}_{2} \times \mathbb{Z}_{2}$ be a Noetherian ring with nontrivial zero-divisors. The following conditions are equivalent:

(1) $\operatorname{diam}(R)=2$

(2) $\operatorname{diam}(R[x])=2$

(3) $\operatorname{diam}\left(R\left[x_{1}, x_{2}, \cdots, x_{n}\right]\right)=2$ for all $n>0$

(4) $\operatorname{diam}(R[[x]])=2$

(5) $Z(R)$ is either the union of two disjoint primes, or $Z(R)$ is prime and $(Z(R))^{2} \neq 0$.

Proof. (1) $\Rightarrow(2)$ was proven in Theorem $5,(2) \Rightarrow(3)$ follows by induction, and $(3) \Rightarrow(2)$ is trivial. (1) $\Rightarrow(4)$ was proven in Theorem 4 . $(1) \Rightarrow(5)$ follows from the above results. We will show that $(2) \Rightarrow(1)$, $(4) \Rightarrow(1)$, and $(5) \Rightarrow(1)$. 
For $(2) \Rightarrow(1)$ and $(4) \Rightarrow(1)$, assume that $\operatorname{diam}(R) \neq 2$. By Theorem 3 , if $\operatorname{diam}(R)=1$ then $\operatorname{diam}(R[x])=1$ since $R \neq \mathbb{Z}_{2} \times \mathbb{Z}_{2}$. The remaining steps have already been commented on at the beginning of this section.

For $(5) \Rightarrow(1)$, first suppose that $Z(R)$ is the union of two disjoint primes $\mathcal{P}_{1}$ and $\mathcal{P}_{2}$. Denote respective elements by $p_{1}$ and $p_{2}$. Assume $a, b \in Z^{*}(R)$ are distinct. If $a, b \in \mathcal{P}_{1}$, then $a b \neq 0$ since $a b \notin \mathcal{P}_{2}$ (so the diameter is at least 2). Since $a p_{2}=0=b p_{2}$, we have a path of length 2. If $a$ and $b$ are in distinct prime ideals then they annihilate each other since the two primes are disjoint.

If $Z(R)$ is prime, Theorem 82 of [5] shows that there is a $z \in Z(R)$ such that $z$ annihilates $Z(R)$. For all $a, b \in Z(R)$ it is clear that $a z=0=b z$ so the diameter is at most 2 . Since $(Z(R))^{2} \neq 0$, Theorem 2.8 of [2] gives $\Gamma(R)$ is not complete, which assures that the diameter is at least 2 . This completes the proof.

Proposition 5. Let $R$ be a commutative ring. If for some $n \in \mathbb{Z}$ with $n>2,(Z(R))^{n}=0$ then $\operatorname{diam}(\Gamma(R[[x]]))=\operatorname{diam}(\Gamma(R[x]))=$ $\operatorname{diam}(\Gamma(R))=2$.

Proof. By hypothesis and Theorem 2.8 of [2], $\Gamma(R)$ is not complete. Hence, there exist distinct $a, b \in Z(R)$ with $a b \neq 0$. Since $(Z(R))^{n-1} \neq$ 0 , there exist $c_{1}, c_{2}, \ldots, c_{n-1} \in Z(R)$ with $c=\prod_{i=1}^{n-1} c_{i} \neq 0$. However, $a c=0=b c$ so $\operatorname{diam}(\Gamma(R))=2$. Let $f(x), g(x) \in Z^{*}(R[[x]])$. By Lemma 1 we have that all coefficients of $f(x)$ and $g(x)$ are elements of $Z(R)$. Thus either $f-g$ or $f-c-g$. So $\operatorname{diam}(\Gamma(R[[x]]))=2$, and the proof is complete.

If $(Z(R))^{2}=0$, then by Theorem 3 we get that each of the three rings has diameter one.

It is still an open question as to whether $R$ a non-Noetherian commutative ring of diameter 2 implies that $R[x]$ is also of diameter 2 , though the work above does provide a partial answer for the cases where $Z(R)=\bigcup_{i \in \Lambda} \mathcal{P}_{i}$ and $\Lambda$ is a finite set. Also unknown is whether rings exist with the properties that the diameter of $R$ and $R[[x]]$ are both 2 while the diameter of $R[x]$ is 3 ; if $R$ is Noetherian, this is not possible as the above work shows. Also, we conjecture if $R$ contains no nilpotent elements, then it is also not possible.

\section{GiRTh OF $\Gamma(R), \Gamma(R[x])$, AND $\Gamma(R[[x]])$}

Now we ask the following question: If $f$ and $g$ are distinct, nonconstant polynomials in $R[x]$ with $f g=0$, does there exists a cycle involving $f$ and $g$ that has at least one vertex contained in $\Gamma(R)$ ? 
Proposition 6. Let $R$ be a commutative ring not necessarily with identity. If $f, g \in Z^{*}(R[x])$ are distinct, nonconstant polynomials with $f g=0$, then there exist $a, b \in Z^{*}(R)$ such that $a-b-g-f-a$ is $a$ cycle in $\Gamma(R[x])$, or $b-f-g-b$ is a cycle in $\Gamma(R[x])$.

Proof. Recall that if $f, g \in Z^{*}(R[x])$, then there exist $a, b \in Z^{*}(R)$ such that $a f=0=b g$. So, if $f g=0$, then we have $a-f-g-b$ is a path. If $a b=0$, then $a-f-g-b-a$ is a cycle.

If $a b \neq 0$, but $b f=0$, then $b-f-g-b$ is a cycle.

If both $a b \neq 0$ and $b f \neq 0$, let $f=c_{0}+c_{1} x+c_{2} x^{2}+\cdots+c_{n} x^{n}$. Then for some $j$ we have $b c_{j} \neq 0$. Then, $a-f-g-b c_{j}-a$ is a cycle.

Observe that if $f \in Z^{*}(R[x])$ with $f$ nonconstant then we can always find a nonconstant polynomial $g \in R[x]$ such that $f \neq g$ and $f g=0$ by letting $g(x)=a x^{1+\operatorname{deg}(f)}$ where $a f=0$. Also note that there always exists some nonconstant $f \in Z^{*}(R[x])$ as long as $Z^{*}(R) \neq \emptyset$. Thus we get the following result.

Corollary 2. If $f \in Z^{*}(R[x])$ a nonconstant polynomial, then there exists a cycle of length 3 or 4 in $\Gamma(R[x])$ with $f$ as one vertex and some $a \in Z^{*}(R)$ as another.

If $Z^{*}(R) \neq \emptyset$, then $\mathrm{g}(\Gamma(R[x])), \mathrm{g}(\Gamma(R[[x]]))<\infty$. We will soon show that $\mathrm{g}(\Gamma(R[x]))=\mathrm{g}(\Gamma(R[[x]]))$.

The preceding paragraph shows that $\Gamma(R[x])$ will always have cycles if $R$ contains nonzero zero-divisors, and there is no upper bound on the length of cycles in $\Gamma(R[x])$.

Proposition 6 is not true in general for $\Gamma(R[[x]])$, as Example 1 shows. However, this proposition will hold for $\Gamma(R[[x]])$ if $R$ is Noetherian. Also, in the proof of Proposition 6, we see there could be a cycle of length 3 in $\Gamma(R[x])$. This is not always the case. If $R=\mathbb{Z} \times \mathbb{Z}$, then $\mathrm{g}(\Gamma(R))=\mathrm{g}(\Gamma(R[x]))=4$. It is not true in general that for any ring $R$ we have $\mathrm{g}(\Gamma(R))=\mathrm{g}(\Gamma(R[x]))$, since if $R=\mathbb{Z}_{4}$, then $\mathrm{g}(\Gamma(R))=\infty$, but $\mathrm{g}(\Gamma(R[x]))=3$. Things improve if $\Gamma(R)$ contains a cycle.

Theorem 7. Let $R$ be a commutative ring, not necessarily with identity. Then $\mathrm{g}(\Gamma(R)) \geq \mathrm{g}(\Gamma(R[x]))=\mathrm{g}(\Gamma(R[[x]]))$. In addition, if $R$ has no nilpotents and $\Gamma(R)$ contains a cycle, then $\mathrm{g}(\Gamma(R))=\mathrm{g}(\Gamma(R[x]))=$ $\mathrm{g}(\Gamma(R[[x]]))$.

Proof. If $Z^{*}(R)=\emptyset$, then $\mathrm{g}(\Gamma(R))=\mathrm{g}(\Gamma(R[x]))=\mathrm{g}(\Gamma(R[[x]]))=\infty$. So we assume $Z^{*}(R) \neq \emptyset$. Since $\Gamma(R)$ is a subgraph of $\Gamma(R[x])$, which is a subgraph of $\Gamma(R[[x]])$, we get that $\mathrm{g}(\Gamma(R)) \geq \mathrm{g}(\Gamma(R[x])) \geq$ $\mathrm{g}(\Gamma(R[[x]]))$. By Corollary 2, $\mathrm{g}(\Gamma(R[x])) \leq 4$. Thus, for the first part of the theorem it suffices to show that if $\mathrm{g}(\Gamma(R[[x]]))=3$, then 
$\mathrm{g}(\Gamma(R[x]))=3$. Assume $\mathrm{g}(\Gamma(R[[x]]))=3$ and that $R$ contains no nilpotent elements. Let $f-g-h-f$ be a cycle in $\Gamma(R[[x]])$. Clearly the coefficients on the lowest degree terms of $f, g$, and $h$ (say $a, b, c$ ) are distinct and form the cycle $a-b-c-a$. This is a cycle of length 3 in $\Gamma(R[x])$ (and in $\Gamma(R)$ ). If $R$ contains a nilpotent element $y$ such that $y^{2}=0$, then $y-y x-y x^{2}-y$ is a cycle of length 3 in $\Gamma(R[x])$. In either case, $\mathrm{g}(\Gamma(R[x]))=3$.

To see the second part of the theorem, observe that if $\mathrm{g}(\Gamma(R[x]))=4$, then $\mathrm{g}(\Gamma(R))=4$ since $\Gamma(R)$ contains a cycle. If $\mathrm{g}(\Gamma(R[x]))=3$, then the result follows from the work above.

Corollary 3. If $R$ contains no nilpotent elements and $\mathrm{g}(\Gamma(R[x]))=3$, then $\mathrm{g}(\Gamma(R))=3$.

Example 2. Consider the ring $\mathbb{Z}_{6}$. This ring has no nilpotent elements and contains no cycle; hence $\mathrm{g}\left(\Gamma\left(\mathbb{Z}_{6}[x]\right)\right)=4$ by Corollary 3.

Observe that Example 2 shows that if $R$ contains no nilpotent elements and $\mathrm{g}(\Gamma(R[x]))=4$ then we cannot say that $\mathrm{g}(\Gamma(R))=4$.

It is not true in general that if $\Gamma(R)$ contains a cycle then $\mathrm{g}(\Gamma(R))=$ $\mathrm{g}(\Gamma(R[x]))$. Let $R=\mathbb{Z} \times \mathbb{Z}_{4}$. Then since $(0,2)-(0,2) x-(0,2) x^{2}-(0,2)$ is a cycle of length three, we have $\mathrm{g}(\Gamma(R[x]))=3$. It is straightforward to verify that $\mathrm{g}(\Gamma(R))=4$.

\section{REFERENCES}

[1] D.D. Anderson, M. Naseer, Beck's coloring of a commutative ring, J. Algebra 159 (1993), 500-514.

[2] D.F. Anderson, P.S. Livingston, The zero-divisor graph of a commutative ring, J. Algebra 217 (1999), 434-447.

[3] I. Beck, Coloring of commutative rings, J. Algebra 116 (1988), 208-226.

[4] D.E. Fields, Zero-divisors and nilpotent elements in power series, Proc. Amer. Math. Soc. 27(3) (1971), 427-433.

[5] I. Kaplansky, Commutative Rings, Univ. of Chicago Press, Chicago, rev. ed., 1974.

[6] N.H. McCoy, Remarks on divisors of zero, Amer. Math. Monthly 49 (1942), 286-295.

[7] S.B. Mulay, Cycles and symmetries of zero-divisors, Comm. Alg. 30(7) (2002), 3533-3558. 
Dept. of Mathematics and Comp. Sci., Wabash College, CrawFORDSVILLE, INDIANA 47933

E-mail address: axtellm@wabash.edu

Department of Mathematics, North Dakota State University, Fargo, NORTh DAKOTA 58105

E-mail address: jim.coykendall@ndsu.nodak.edu

Department of Mathematics, University of Evansville, Evansville, INDIANA 47722

E-mail address: js298@evansville.edu 